\title{
Fiber-Optic Bend Sensor Based on Double Cladding Fiber
}

\author{
Oleg V. Ivanov ${ }^{1,2,3}$ and Alexey A. Chertoriyskiy ${ }^{1,3}$ \\ ${ }^{1}$ Ulyanovsk Branch of Kotel'nikov Institute of Radio Engineering and Electronics of Russian Academy of Sciences, \\ Ulitsa Goncharova 48, Ulyanovsk 432011, Russia \\ ${ }^{2}$ Ulyanovsk State University, Ulitsa L. Tolstogo 42, Ulyanovsk 432017, Russia \\ ${ }^{3}$ Ulyanovsk State Technical University, Severny Venetz Street 32, Ulyanovsk 432027, Russia
}

Correspondence should be addressed to Oleg V. Ivanov; olegivvit@yandex.ru

Received 31 March 2015; Revised 18 June 2015; Accepted 24 June 2015

Academic Editor: Christos Riziotis

Copyright (c) 2015 O. V. Ivanov and A. A. Chertoriyskiy. This is an open access article distributed under the Creative Commons Attribution License, which permits unrestricted use, distribution, and reproduction in any medium, provided the original work is properly cited.

\begin{abstract}
We develop and investigate fiber-optic bend sensor, which is formed by a section of double cladding SM630 fiber between standard SMF-28 fibers. The principle of operation of the sensor is based on coupling of the fiber core and cladding modes at the splices of fibers having different refractive index profiles. We use two sources with wavelengths 1328 and $1545 \mathrm{~nm}$ to interrogate the sensor. The dependences of transmission on curvature at these wavelengths are significantly different. We show that the proposed sensor is able to perform measurements of curvature with radii from meters to $26 \mathrm{~cm}$ with accuracy of about $3 \%$.
\end{abstract}

\section{Introduction}

A wide class of fiber-optic sensors is based on the use of cladding modes of the fiber. Such sensors are utilized for measurements of temperature, various types of deformations, refractive index and chemical composition of the medium, and other parameters [1-3]. The principle of operation of these sensors is that the cladding modes are highly sensitive to influences exerted on the fiber guiding the cladding modes. In addition, the cladding modes are sensitive to parameters of the external medium, such as refractive index and absorption (amplification) coefficient. This sensitivity is caused by propagation of the evanescent field of the cladding modes along the fiber cladding surface having contact with the external medium.

To use fiber cladding modes in a sensor, these modes should be excited by transferring energy from the core mode that comes from the fiber line. The excitation can be realized by selective resonance coupling for one mode at a particular wavelength or without resonance for many modes in the whole spectral range. For the resonance excitation, the longperiod fiber gratings and, in some cases, Bragg gratings are used. In order to excite cladding modes without resonance, defects of fiber structure disturbing propagation of the core mode are created [4-6].

If a cascade of two defects is created in one fiber, an interferometric structure is formed. In such a structure, the light interferes after propagation through the core and the cladding, which act like two arms of an interferometer [7, 8]. For example, there are studied interferometers with a longperiod fiber grating $[4,5]$, misaligned splicing point $[4,9,10]$, a nonadiabatic taper [11], a photonic crystal fiber having collapsed air holes [5], and splice of fibers with unmatched mode profiles (single-mode/multimode fiber splice) [12-15]. These structures find application in sensors of strain, temperature $[16,17]$, and bend [18] and also in multiwavelength fiber laser [19]. In fibers with two defects, effects of mode coupling are observed along with the interference effect [20-22].

Earlier we proposed a new structure that was based on a section of SM630 fiber with double cladding between standard fibers $[23,24]$. Wide loss bands were found in the transmission spectrum of the investigated structure. These bands are caused by core-cladding mode coupling at wavelengths of convergence of the propagation constants of fiber modes $[20,21]$. It was shown that the wavelengths of the bands depended on curvature of the fiber [24]. In this 


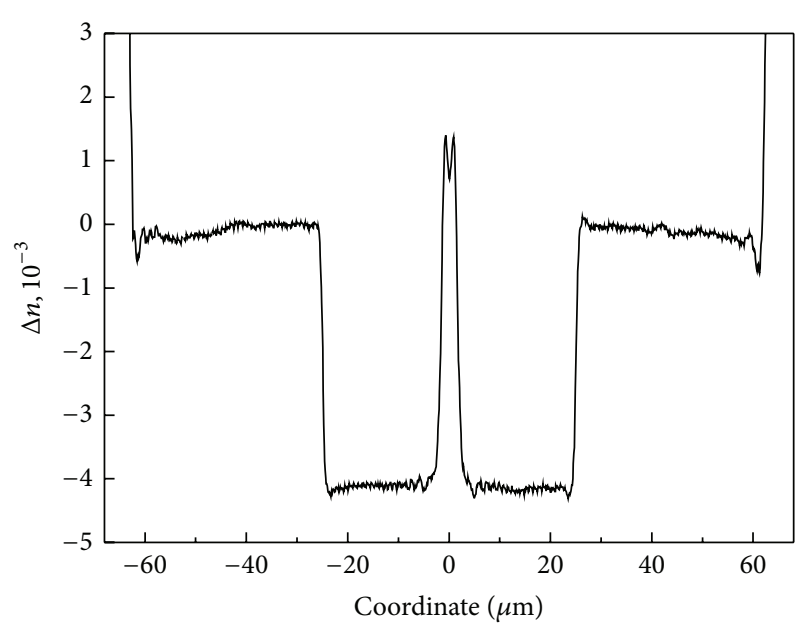

FIgURE 1: Refractive index profile of SM630 fiber.

paper, we describe a bend sensor utilizing this effect. The interrogation is realized by comparison between transmissions at two different wavelengths. As light sources we use light diodes with standard telecommunication wavelengths. We investigate the range of curvatures measurable by the designed sensor and the accuracy of measurements.

\section{Double Cladding Fiber Structure}

The fiber structure used in the sensor is formed by a section of SM630 fiber (3M specialty optical fiber) between two standard SMF-28 fibers $\left(r_{\text {co }}=4.2 \mu \mathrm{m}, \Delta=0.36 \%\right.$, NA = $0.14 \%$, and $\lambda_{\text {cutoff }}=1260 \mathrm{~nm}$ ). The fibers are spliced with an ordinary automatic splicer.

SM630 fiber that used an insertion has an inner cladding with refractive index $n_{\text {inn }}$, which is lower than the refractive index of the outer cladding $n_{\mathrm{cl}}: n_{\mathrm{cl}}-n_{\mathrm{inn}}=0.0043$ (Figure 1). The radius of the inner cladding is $25 \mu \mathrm{m}$. The fiber core has radius that is about one half of that of the standard fiber: $r_{\text {co }}=$ $1.8 \mu \mathrm{m}$. The difference between refractive indices of the core $n_{\text {co }}$ and the inner cladding is $n_{\text {co }}-n_{\text {inn }}=0.0054$.

At the first splice of SMF-28 and SM630 fibers, having different parameters of the cores, power from the core mode of SMF-28 fiber is distributed between modes of SM630 fiber as a result of different refractive index profiles. From the first splice, the modes propagate through the section of SM630 fiber to the second splice. Some part of light is scattered due to coupling to radiation modes and high-order modes having high losses at the fiber surface.

At the second splice, the modes of SM630 fiber are coupled to the core mode of SMF-28 fiber, where they interfere. Here, some part of light goes into the cladding of SMF-28 fiber, where it is lost and does not return to the core. The section of SM630 fiber was painted with a black ink, in order to increase losses for high-order modes and eliminate their interference. The scheme of propagation of light in the structure is shown in Figure 2.

Investigation of the structure presented earlier [23] has shown that its spectrum exhibits two main dips at wavelengths 1185 and $1450 \mathrm{~nm}$. It has also been demonstrated that

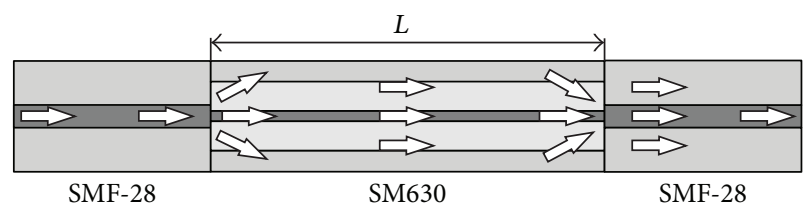

FIGURE 2: Scheme of propagation of light in the fiber structure.

the spectra of the fiber structure depend strongly on bending of the fiber insertion [25]. So, when the curvature increases, the dips shift to long wavelengths and increase their depth. In Figure 3, we show (a) the full spectrum and (b) its evolution in two bands from 1300 to $1350 \mathrm{~nm}$ and from 1530 to $1580 \mathrm{~nm}$ for the structure of length $14 \mathrm{~cm}$ when the curvature radius changes from infinity to $23 \mathrm{~cm}$. We choose these wavelength bands due to their use in telecommunication and availability of fiber sources at these wavelengths.

The dips in the spectrum are formed at wavelengths where the propagation constants of two modes converge, which is possible due to the fact that the fiber external cladding has refractive index higher than the refractive index of the inner cladding [24]. When the fiber is bent, the refractive index changes inhomogeneously both in the core and in the claddings. The change of refractive index profile results in a shift of mode fields that change their propagation constants. As a result, the modes that converged at one wavelength now converge at a different wavelength. This would appear in the spectrum as a shift of the dips in the bent fiber.

\section{Fiber-Optic Bend Sensor}

The investigated structure is easy to fabricate and contains only optical fibers and no other elements; therefore, the shift of dips under bending can be used to create an intrinsic fiber sensor, which should be durable in harsh environment such as high temperature and radiation, with the same durability as the fiber that is employed in the sensor.

Various interrogation schemes of the sensor can be suggested, starting with the scheme that reads the full spectrum and measures the shift of the dip under bending. However, this method requires an expensive spectrum analyzer. It is also possible to do measurements with two light sources at different wavelengths, when transmission at one wavelength depends on the fiber curvature, while the transmission at other wavelengths is an independent reference signal.

Because of availability of telecommunication light sources with working wavelengths around 1310 и $1550 \mathrm{~nm}$, we designed a scheme with two sources at these wavelengths. The transmission at a wavelength of $1310 \mathrm{~nm}$ is a reference (it depends weakly on bending of the fiber), and the signal at a wavelength of $1550 \mathrm{~nm}$ is the main carrier of information about curvature (as is seen from Figure 3(b), transmission at this wavelength decreases by $20 \mathrm{~dB}$ when the fiber is bent). The controller is used for channel switching and synchronous reading of the detector, which receives the light coming from one of the sources through coupler and the sensor head (see Figure 4). 

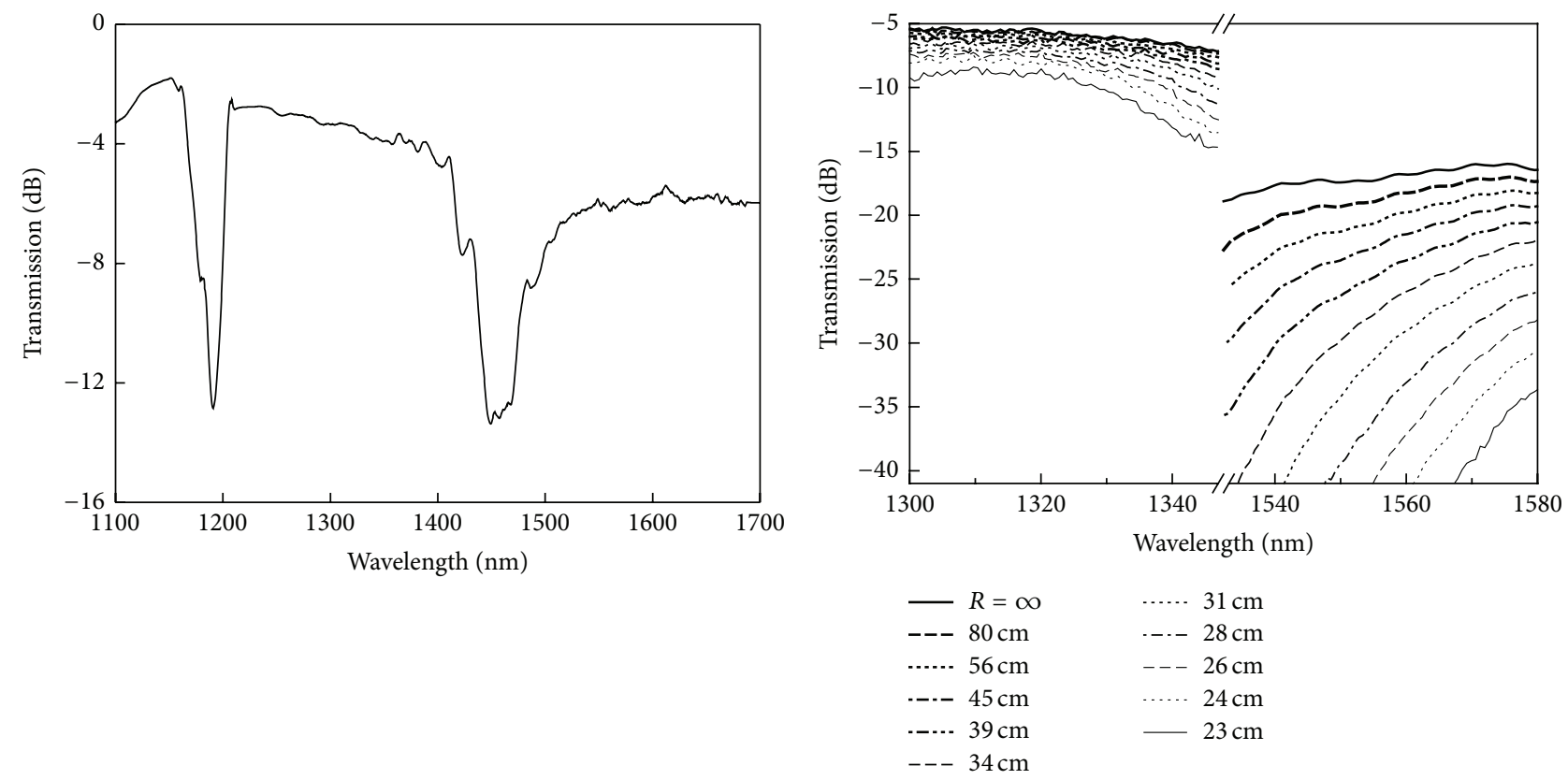

(a)

(b)

FIGURE 3: (a) Full spectrum of the structure and (b) evolution of spectrum in two working bands for curvature radius changing from infinity to $23 \mathrm{~cm}$.

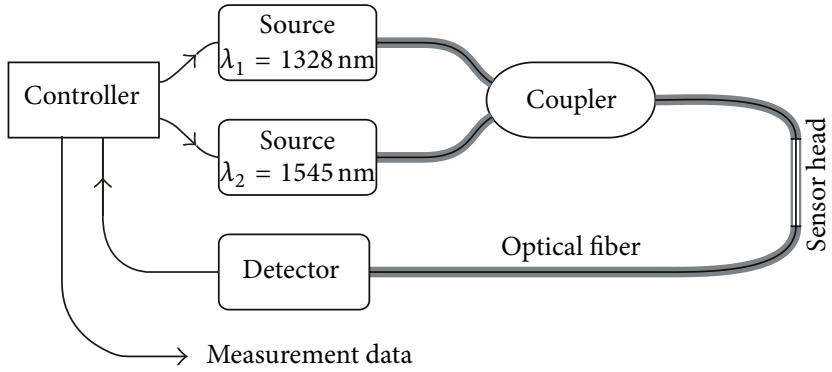

FIGURE 4: Interrogation scheme of the fiber bend sensor.

In Figure 5, the spectra of the sources are shown. Each spectrum contains 5 major peaks around central wavelengths 1328 and $1545 \mathrm{~nm}$ with wavelength distance between peaks 0.6 and $0.8 \mathrm{~nm}$, respectively, and envelopes having widths 2.4 and $3.5 \mathrm{~nm}$. The presence of several spectral peaks does not prevent from using these sources, since the spectral inhomogeneities of the fiber structure (which have widths of order of $50 \mathrm{~nm}$ ) are much wider than the distance between the peaks of the sources.

To make a bend along a fixed curvature radius we inserted the fiber inside a flexible polymer tube, which was attached at the edge of an elastic metal plate of length $50 \mathrm{~cm}$. The section of double cladding fiber of length $14 \mathrm{~cm}$ was placed in the middle of the metal plate; therefore, when the plate was bent, the fiber deformation was symmetric. The ends of the plate were set against two posts, one of which was movable by a screw along a scale and could squeeze the plate. Thus the plate was curved (Figure 6).

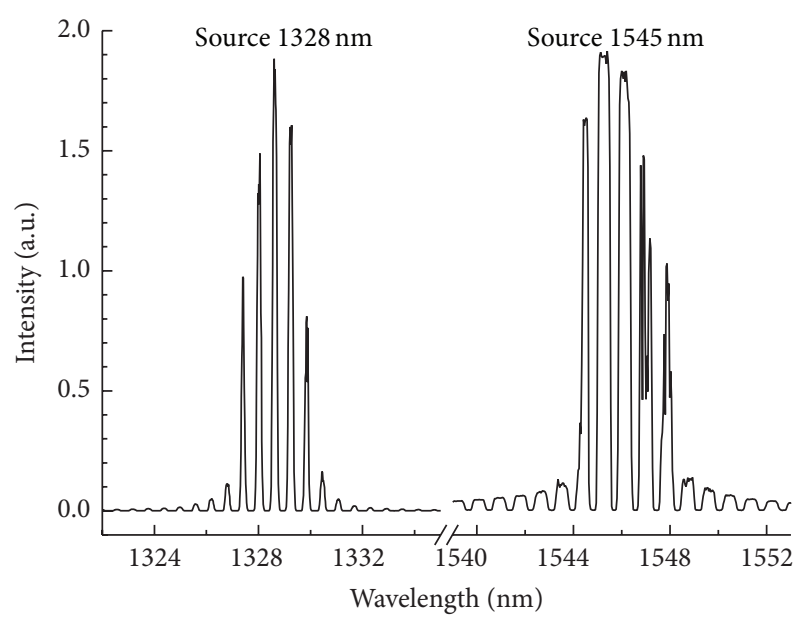

FIgURE 5: Spectra of the sources used in the experiment.

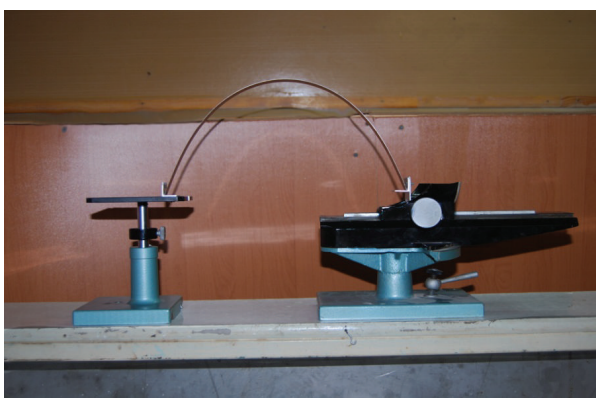

Figure 6: The mount for fiber structure bending. 


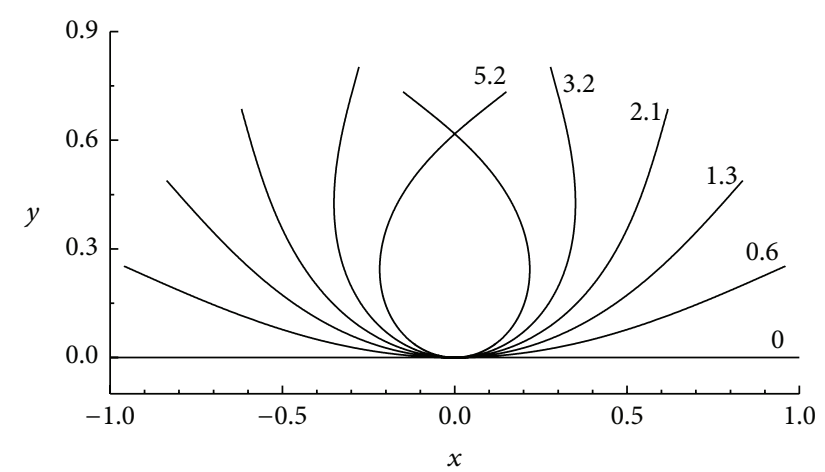

FIGURE 7: Change of the plate shape when its ends are brought together. The numbers near the curves indicate the curvature value in the coordinate origin.

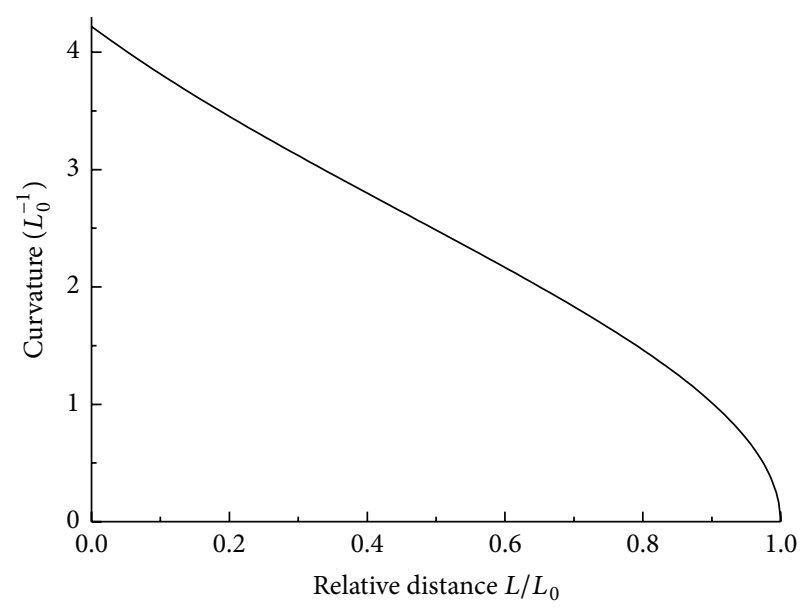

FIGURE 8: Dependence of curvature in the plate center on the relative distance between the ends.

Bending of the plate with loose ends is described by Jacobi elliptic functions, which are the solution of the equation of mathematics pendulum $d^{2} \theta / d s^{2}=\sin \theta$, where $\theta$ is the angle of the curve and $s$ is distance along the curve. Figure 7 shows the curves that are the solutions of the given equation and demonstrates the change of the plate shape when its ends are drawn together. Different curves correspond to different values of curvature in the coordinate origin. When the curvature becomes higher than 2.8 , the ends of the plate start to bend inwards; the branches intersect for curvatures above 4.2 .

For moderate bends, the curvature in the middle of the plate is close to a constant and is unambiguously determined by the distance between the ends of the plate. The dependence of curvature in the plate center on relative decrease of the distance between the ends is given in Figure 8. When the ends clamp, the curvature radius in the center is 0.238 of the plate length. When the ends are separated, the curvature goes to zero.

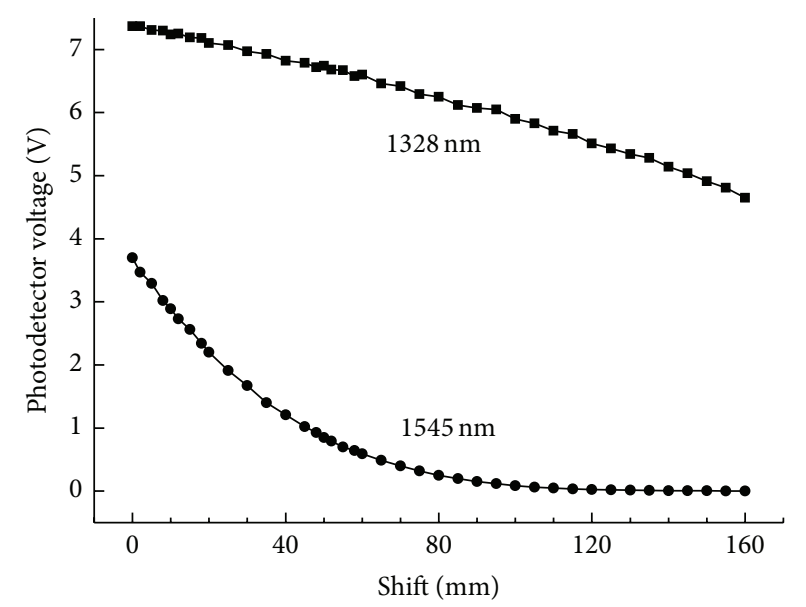

FIGURE 9: Dependence of transmission on the shift of one end of the plate at two wavelengths.

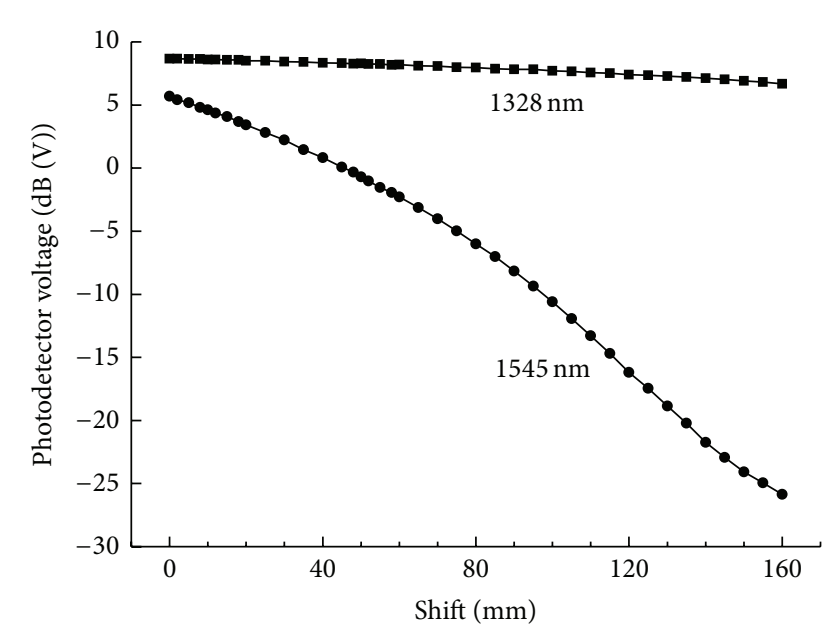

FIGURE 10: Logarithmic dependence of photodetector voltage on shift of the end of the plate at two wavelengths.

\section{Experimental Results}

The measurements of intensity of light transmitted through the fiber-optic system were started for the straight plate, when transmission at both wavelengths is maximal. After the movable post was shifted closer to the fixed post, the metal plate bent together with the fiber section of the sensor. As a result, the transmitted intensity decreased. By measuring transmission at two wavelengths and switching the sources at $1328 \mathrm{~nm}$ and $1545 \mathrm{~nm}$, we obtained the dependence of transmission on the shift of the post (Figure 9). This shift can be related to curvature using Figure 8 . The maximum shift of $160 \mathrm{~mm}$ corresponds to a relative distance $L / L_{0}=0.68$, which gives curvature $1.9 L_{0}^{-1}$ and a curvature radius of $26 \mathrm{~cm}$.

For light at a wavelength of $1328 \mathrm{~nm}$, the change of transmitted intensity is close to a linear dependence and the maximum decrease of the signal is about $30 \%$. The amplitude of the signal at $1545 \mathrm{~nm}$ depends exponentially on the shift of the post and decreases by three orders of magnitude at maximum curvature. This is seen in Figure 10, where 


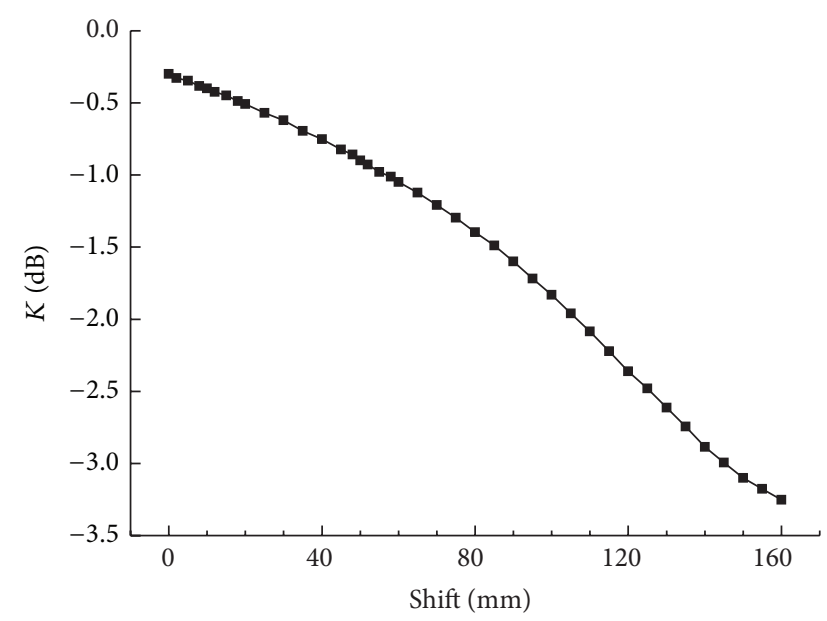

FIGURE 11: Relation between signals at two wavelengths as a function of the plate bending.

the voltage of the photodetector (related to $1 \mathrm{~V}$ ) is shown in logarithmic scale.

To eliminate the influences of intensity changes that are unrelated to bending of the sensing section of the fiber and are independent of wavelength, we introduce the following function $K$ describing bending, which is defined as relation between transmissions at $1328 \mathrm{~nm}$ and $1545 \mathrm{~nm}$ :

$$
K=\frac{T(1545 \mathrm{~nm})}{T(1328 \mathrm{~nm})} \text {. }
$$

In Figure 11, we show the dependence of the relation $K$ on bending of the plate. It is seen from the figure that this relation decreases monotonically upon the shift of the plate end. This fact allows us to use that relation as a value determining the curvature of the fiber. The dependence is rather smooth; therefore, we may expect small random error in the measured curvature value.

In order to examine the stability of work of the sensor, we compared the dependences for increasing and decreasing bending of the plate. The results of measurements are given in Figure 12. The full cycle of bending and unbending of the plate resulted in a minor change of the value of $K$. As shown in subsequent analysis, this change is caused by instability of the light sources, whose intensity changed with time. Hysteresis effects in the sensor head itself have not been observed. After one cycle of bending and unbending we obtain a new value of $K$, which corresponds now to a nonzero shift of the plate and nonzero curvature. From Figure 12, the change of the shift is about $6 \mathrm{~mm}$. By dividing this change by the maximum shift of $160 \mathrm{~mm}$, we estimate the accuracy of measurements of curvature to have a magnitude of about $3 \%$.

\section{Conclusion}

Thus, we have shown that the fiber structure based on a section of double cladding fiber is sensitive to bending. Transmission through the structure at 1328 and $1545 \mathrm{~nm}$ depends differently on curvature of the fiber, which has

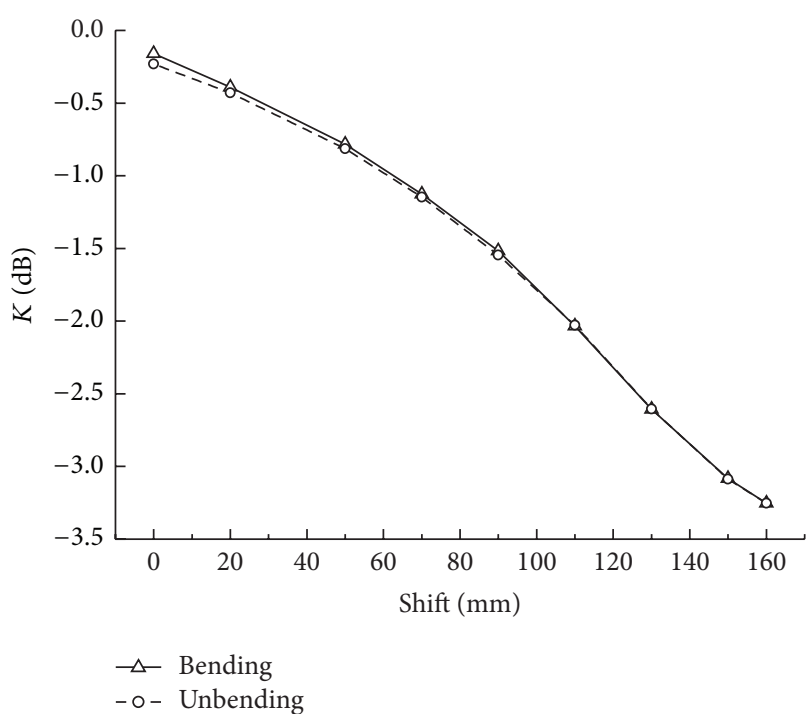

FIGURE 12: Relation between two voltages during bending and unbending of the plate.

allowed us to create a fiber-optic bend sensor using two light sources with corresponding wavelengths. Transmission at $1545 \mathrm{~nm}$ decreases exponentially with curvature of the fiber, while transmission at $1328 \mathrm{~nm}$ changes insignificantly. Therefore, the first value is used in the designed sensor as a signal, and the second as a reference. The dimensions of the sensor head are $1 \times 1 \times 100 \mathrm{~mm}$. The range of curvatures that can be measured by the sensor is from meters to $26 \mathrm{~cm}$.

\section{Conflict of Interests}

The authors declare that there is no conflict of interests regarding the publication of this paper.

\section{Acknowledgment}

This work was supported by the Ministry of Education and Science of the Russian Federation (Project no. 14.Z50.31.0015).

\section{References}

[1] O. V. Ivanov, S. A. Nikitov, and Y. V. Gulyaev, "Cladding modes of optical fibers: properties and applications," Physics-Uspekhi, vol. 49, no. 2, pp. 167-191, 2006.

[2] S. W. James and R. P. Tatam, "Optical fibre long-period grating sensors: characteristics and application," Measurement Science and Technology, vol. 14, no. 5, pp. R49-R61, 2003.

[3] A. Lim, W. B. Ji, and S. C. Tjin, "Improved refractive index sensitivity utilizing long-period gratings with periodic corrugations on cladding," Journal of Sensors, vol. 2012, Article ID 483471, 5 pages, 2012.

[4] X. Dong, L. Su, P. Shum, Y. Chung, and C. C. Chan, "Wavelength-selective all-fiber filter based on a single longperiod fiber grating and a misaligned splicing point," Optics Communications, vol. 258, no. 2, pp. 159-163, 2006. 
[5] H. Y. Choi, K. S. Park, and B. H. Lee, "Photonic crystal fiber interferometer composed of a long period fiber grating and one point collapsing of air holes," Optics Letters, vol. 33, no. 8, pp. 812-814, 2008.

[6] L.-L. Xue and L. Yang, "Sensitivity enhancement of RI sensor based on SMS fiber structure with high refractive index overlay," Journal of Lightwave Technology, vol. 30, no. 10, pp. 1463-1469, 2012.

[7] F. Gu, X. Zhang, J. Xiong, F. Pang, and T. Wang, "Fabrication and simulation of cascaded Fabry-Perot micro-cavities in fibers," in Advanced Sensor Systems and Applications VI, vol. 9274 of Proceedings of SPIE, October 2014.

[8] B. H. Lee, Y. H. Kim, K. S. Park et al., "Interferometric fiber optic sensors," Sensors, vol. 12, no. 3, pp. 2467-2486, 2012.

[9] J. Canning and A. L. G. Carter, "Modal interferometer for in situ measurements of induced core index change in optical fibers," Optics Letters, vol. 22, no. 8, pp. 561-563, 1997.

[10] X. Yu, P. Shum, and X. Dong, "Photonic-crystal-fiber-based Mach-Zehnder interferometer using long-period gratings," Microwave and Optical Technology Letters, vol. 48, no. 7, pp. 1379-1383, 2006.

[11] O. Frazão, R. Falate, J. L. Fabris, J. L. Santos, L. A. Ferreira, and F. M. Araújo, "Optical inclinometer based on a single long-period fiber grating combined with a fused taper," Optics Letters, vol. 31, no. 20, pp. 2960-2962, 2006.

[12] S. Silva, O. Frazão, J. Viegas et al., “Temperature and strain-independent curvature sensor based on a singlemode/multimode fiber optic structure," Measurement Science and Technology, vol. 22, Article ID 085201, 2011.

[13] D.-P. Zhou, L. Wei, W.-K. Liu, Y. Liu, and J. W. Y. Lit, "Simultaneous measurement for strain and temperature using fiber Bragg gratings and multimode fibers," Applied Optics, vol. 47, no. 10, pp. 1668-1672, 2008.

[14] T.-J. Chen, "Use of liquid-crystal-clad fiber as a modal filter for a two-mode fiber-optic interferometer," Optics Letters, vol. 29, no. 24, pp. 2852-2854, 2004.

[15] T.-J. Chen, "A novel two-mode fiber-optic interferometer based on $\mathrm{HE}_{11}-\mathrm{TE}_{01}$ modal interference utilizing a liquid-crystal-clad fiber modal filter," Optics Communications, vol. 261, no. 1, pp. 43-50, 2006.

[16] W. Chen, S. Lou, L. Wang et al., "In-fiber modal interferometer based on dual-concentric-core photonic crystal fiber and its strain, temperature and refractive index characteristics," Optics Communications, vol. 284, no. 12, pp. 2829-2834, 2011.

[17] H. M. Chan, R. Huang, and H. Lee, "A compact Mach-Zehnder two-mode fiber-optic interferometer for high temperature sensing," in Proceedings of the IEEE Conference on Sensors, pp. 621623, November 2005.

[18] Y. Liu and L. Wei, "Low-cost high-sensitivity strain and temperature sensing using graded-index multimode fibers," Applied Optics, vol. 46, no. 13, pp. 2516-2519, 2007.

[19] Q. Zhang, X. Zeng, F. Pang, M. Wang, and T. Wang, "Switchable multiwavelength fiber laser by using a compact in-fiber MachZehnder interferometer," Journal of Optics, vol. 14, no. 14, Article ID 045403, 5 pages, 2012.

[20] F. Pang, W. Xiang, H. Guo et al., "Special optical fiber for temperature sensing based on cladding-mode resonance," Optics Express, vol. 16, no. 17, pp. 12967-12972, 2008.

[21] F. Pang, W. Liang, W. Xiang et al., "Temperature-insensitivity bending sensor based on cladding-mode resonance of special optical fiber," IEEE Photonics Technology Letters, vol. 21, no. 2, pp. 76-78, 2009.
[22] Y. Zhao, F. Pang, Y. Dong, J. Wen, Z. Chen, and T. Wang, "Refractive index sensitivity enhancement of optical fiber cladding mode by depositing nanofilm via ALD technology," Optics Express, vol. 21, no. 22, pp. 26136-26143, 2013.

[23] O. V. Ivanov, "Fibre-optic interferometer formed by a section of small-core fibre spliced between standard fibres," Optics Communications, vol. 282, no. 19, pp. 3895-3898, 2009.

[24] O. V. Ivanov and I. V. Zlodeev, "Fiber structure based on a depressed inner cladding fiber for bend, refractive index and temperature sensing," Measurement Science and Technology, vol. 25, no. 1, Article ID 015201, 8 pages, 2014.

[25] I. V. Zlodeev and O. V. Ivanov, "Transmission spectra of a double-clad fibre structure under bending," Quantum Electronics, vol. 43, no. 6, pp. 535-541, 2013. 

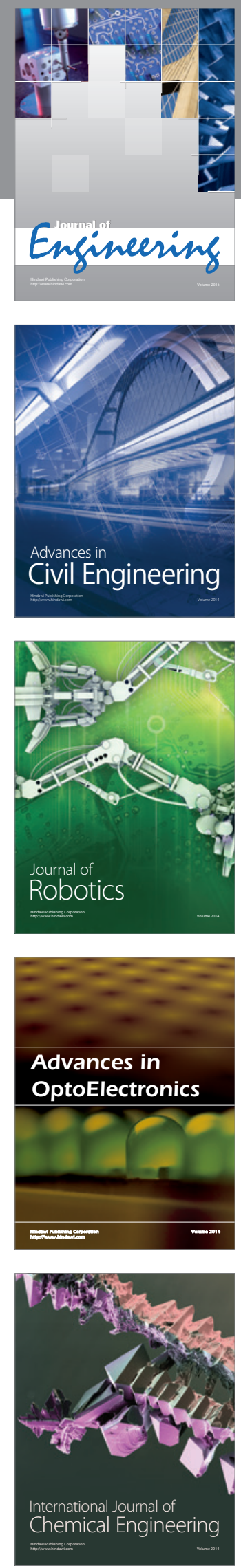

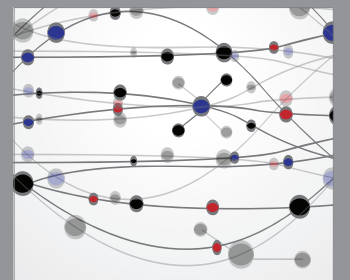

The Scientific World Journal
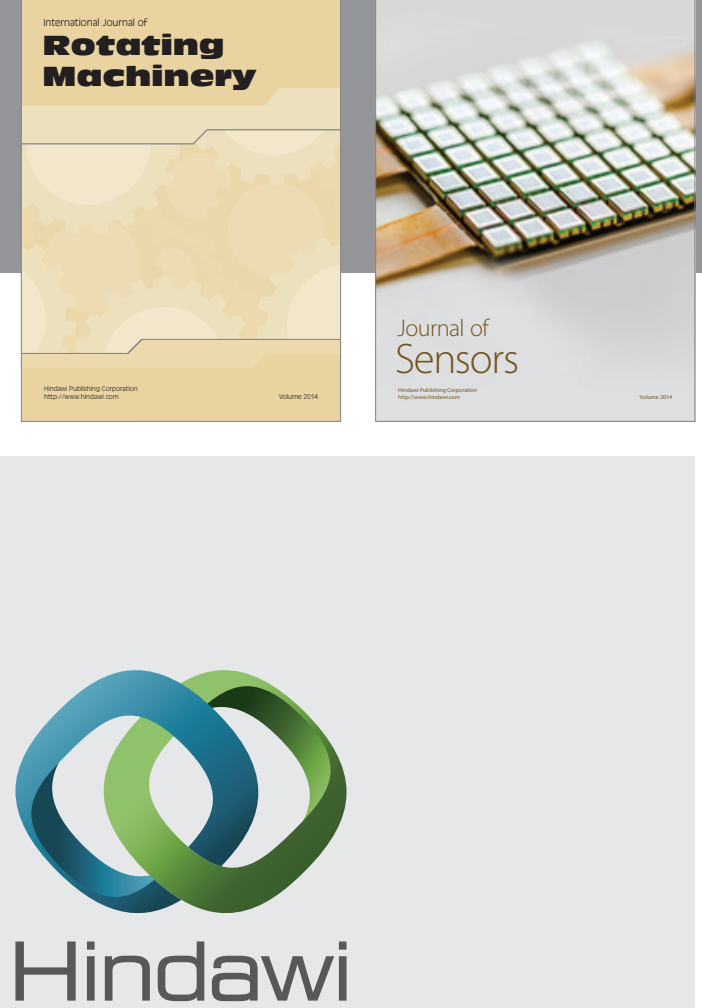

Submit your manuscripts at http://www.hindawi.com
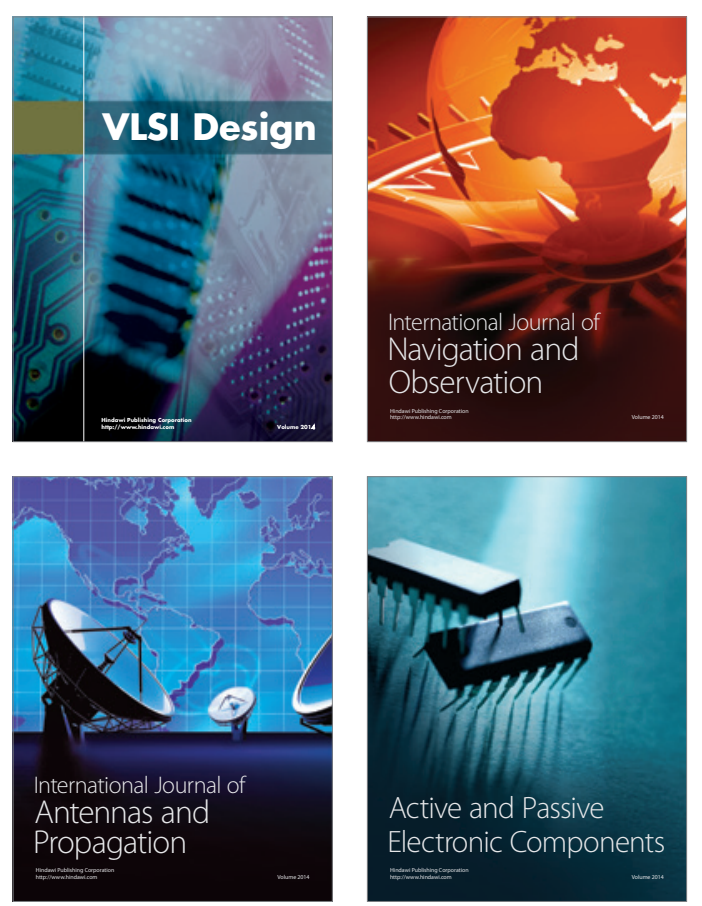
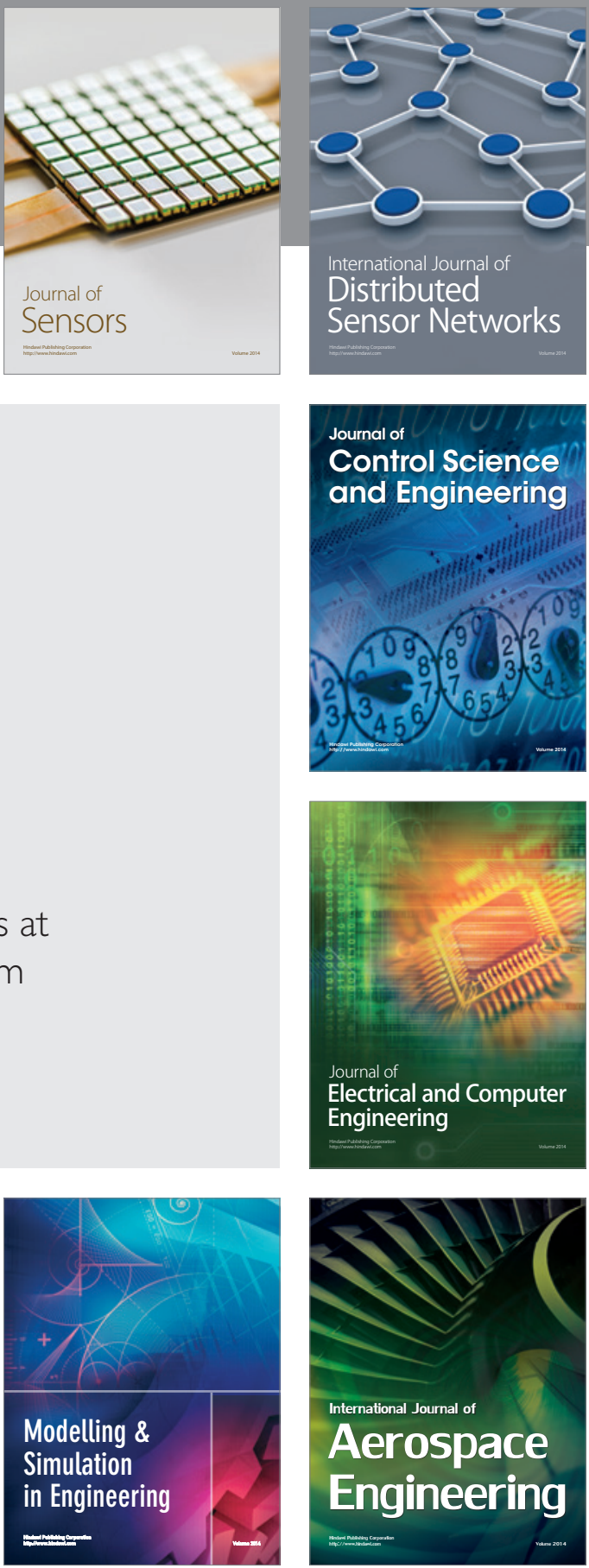

Journal of

Control Science

and Engineering
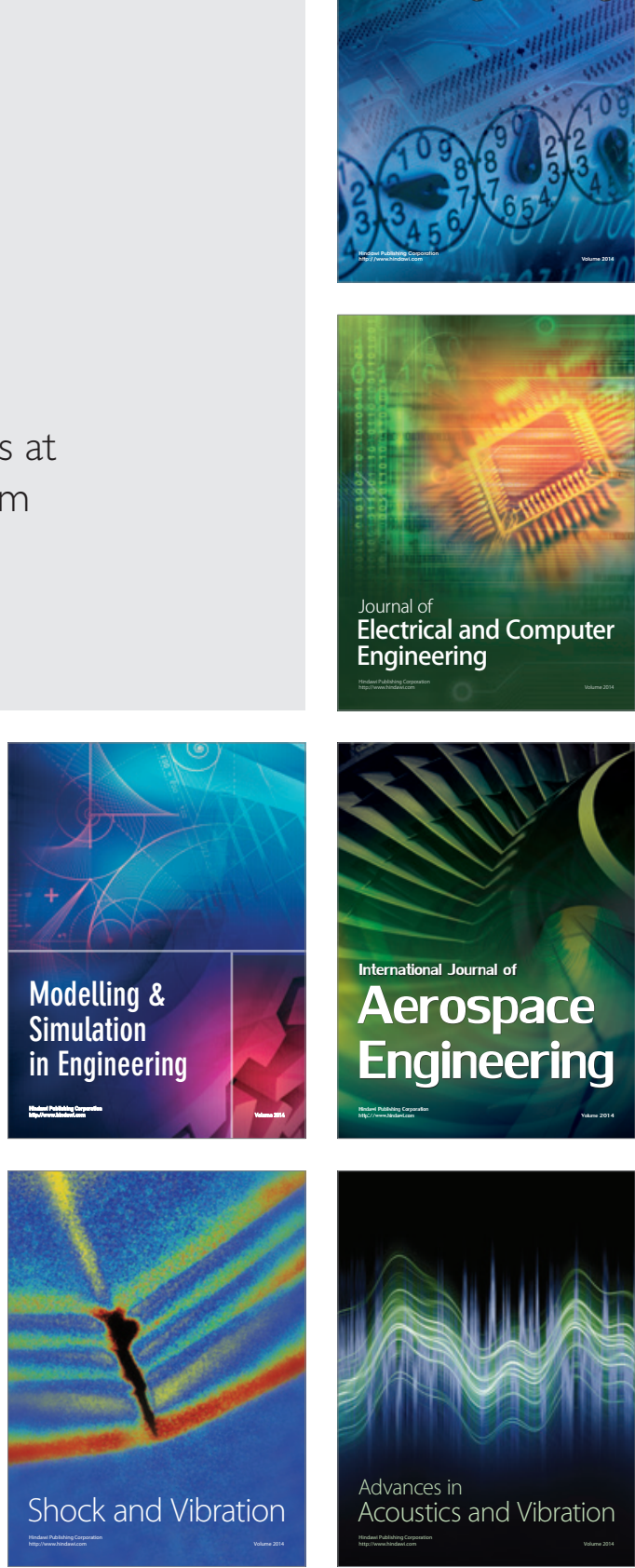